\title{
THE DYNAMICS OF SWALLOWING. II. NEUROMUSCULAR DYSPHAGIA OF PHARYNX
}

\author{
By PHILIP KRAMER, MICHAEL ATKINSON, STANLEY M. WYMAN, AND \\ FRANZ J. INGELFINGER \\ (From the Evans Memorial, Massachusetts Memorial Hospitals, and the Department of Medi- \\ cine, Boston University School of Medicine, and the Massachusetts General Hospital \\ and the Department of Radiology, Harvard Medical School, Boston, Mass.)
}

(Submitted for publication October 5, 1956; accepted December 7, 1956)

Neuromuscular disorders involving the pharyngeal phase of swallowing have hitherto been classified on a radiological basis into descriptive but rather nonspecific categories such as vallecular dysphagia (1), so called on account of the residuum of barium seen after swallowing in the valleculae and pyriform sinuses, spasm of the mouth of the esophagus $(2,3)$ and cricopharyngeal achalasia (4). The exact pathophysiology has been difficult to define using radiological techniques alone, although the development of cinefluoradiography in recent years has enabled detailed observation of the anatomical movements of deglutition. This technique, however, has as yet given little additional information about the nature and extent of the propulsive defect in the neuromuscular dysphagias. In particular, the question of whether functional disturbance of the cricopharyngeus ${ }^{1}$ hinders the passage of the bolus is unsettled.

To investigate disorders of the swallowing mechanisms in patients with various neuromuscular dysphagias, we have applied the same technique of intraluminal pressure recording and simultaneous rapid serial radiography which has been used to investigate normal swallowing (5).

\section{METHODS}

With the patient supine, resting intraluminal pressures were recorded from the pharynx and esophagus by means of an open-ended, metal-tipped polyethylene tube attached to a Sanborn electromanometer. This was passed through the nose until its tip lay in the esophagus and then, under fluoroscopic control, withdrawn centi-

1 The terms cricopharyngeus and cricopharyngeal sphincter are here used to include the entire sphincteric mechanism, composed of striated muscle, and including not only fibers from the inferior constrictor (i.e., the cricopharyngeus) but perhaps also circular muscle from the upper esophagus. meter by centimeter. At each level, resting intraluminal pressure was recorded.

Intraluminal pressures on swallowing were recorded simultaneously by means of three such tubes fastened together so that the distance between the distal and middle tip was $2 \mathrm{~cm}$. and between the middle and proximal tip $4 \mathrm{~cm}$. Whilst pressures were being recorded during barium swallows, serial radiographs at a rate of 6 per second were taken in the anteroposterior and lateral planes. All swallowing studies were made with the patients in the sitting position, as many had difficulty in swallowing when supine.

Patients studied. Seven patients with dysphagia were studied (Table I). Four had had bulbar poliomyelitis, two suffered from myasthenia gravis, and the seventh had dystrophia myotonica. Six patients were studied by simultaneous radiography and pressure recording; only pressure records were obtained in the seventh patient, a 30-year-old male with mild postbulbar dysphagia.

\section{Dysphagia following bulbar poliomyelitis}

Resting intraluminal pressures. Pressures in the lumen of the pharynx and esophagus were within normal limits. At the level of the cricoid cartilage a zone of high resting pressure was found, resembling that present in normal subjects and caused, it is believed, by tonic contraction of the cricopharyngeus $(5,6)$. In the four patients, maximal resting pressures in this high pressure zone were $48,25,30$, and $22 \mathrm{~mm}$. $\mathrm{Hg}$, respectively, figures which correspond well with the mean normal value of $35 \mathrm{~mm}$. $\mathrm{Hg}$. In terms of the pressures recorded, no evidence of either paralysis or hypertonicity of the cricopharyngeus was obtained.

Deglutition pressures (Figure 1). In three of the four subjects hardly any pressure change occurred in the upper or lower pharynx. The initial peak of intrapharyngeal pressure was never present, but in the lower pharynx a slight increase in pressure was occasionally seen at the time the second peak of pressure occurs in normal subjects. In the fourth subject, who had only mild 
TABLE I

Patients studied

\begin{tabular}{|c|c|c|c|c|}
\hline Disease & $\begin{array}{r}\text { Patient' } \\
\text { sex and a }\end{array}$ & & $\begin{array}{l}\text { Duration of } \\
\text { dysphagia }\end{array}$ & Disability at time of study \\
\hline \multirow[t]{4}{*}{$\begin{array}{l}\text { Dysphagia following } \\
\text { poliomyelitis }\end{array}$} & Male & 27 & 7 months & $\begin{array}{l}\text { Nasal voice. Frequent choking on swallowing } \\
\text { and occasional regurgitation through the nose. }\end{array}$ \\
\hline & Female & 32 & 9 months & $\begin{array}{l}\text { Nasal voice. Had required tube feeding for } \\
\text { several months after attack and even at time } \\
\text { of study could only swallow liquids and semi- } \\
\text { solids. }\end{array}$ \\
\hline & Male & 41 & 2 years & Nasal voice. Difficulty in swallowing solids. \\
\hline & Male & 30 & 4 months & $\begin{array}{l}\text { Normal voice. Difficulty in swallowing solids } \\
\text { towards end of meal. }\end{array}$ \\
\hline \multirow[t]{2}{*}{ Myasthenia gravis } & Female & 19 & 2 years & $\begin{array}{l}\text { Nasal voice. Moderate dysphagia relieved by } \\
\text { prostigmine. }\end{array}$ \\
\hline & Male & 65 & 3 months & $\begin{array}{l}\text { Slightly nasal voice. Difficulty in swallowing } \\
\text { partially relieved by prostigmine. }\end{array}$ \\
\hline Dystrophia myotonica & Male & 60 & 5 years & $\begin{array}{l}\text { Nasal voice and dysarthria. Marked dyspha- } \\
\text { gia. Swallowing had to be assisted by tilting } \\
\text { head backwards. }\end{array}$ \\
\hline
\end{tabular}

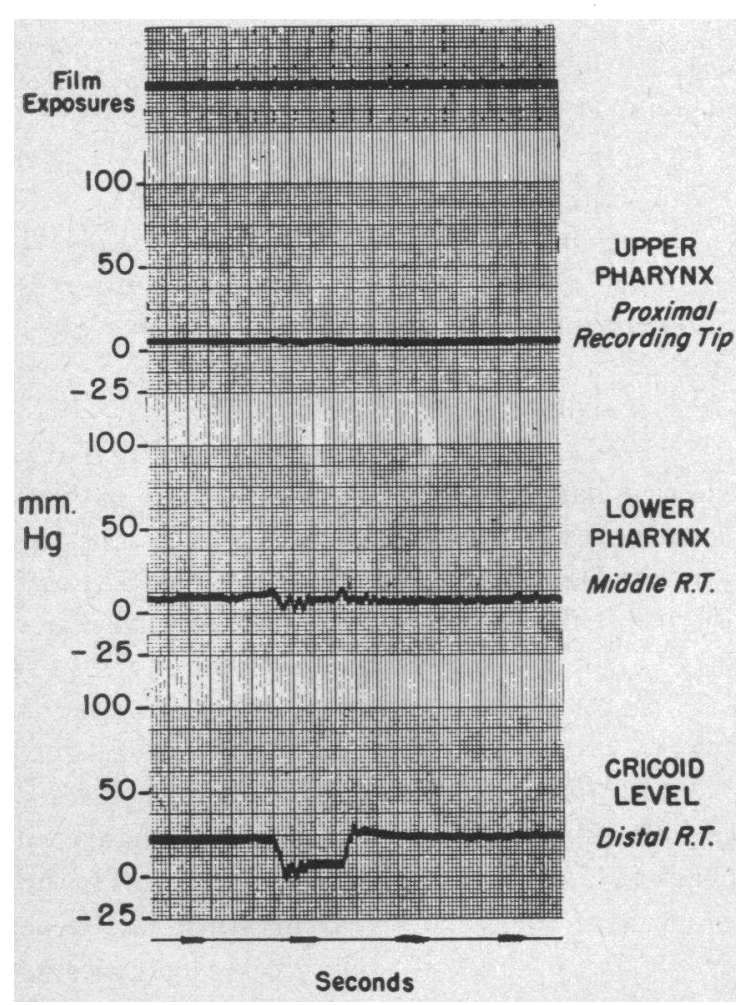

Fig. 1. Deglutition Pressures in a Patient with Dysphagia Following Poliomyelitis

R.T. = recording tip. dysphagia, pressure complexes normal in type but low in amplitude were recorded from the pharynx.

The pressures recorded in the cricopharyngeal area showed a response to swallowing that was normal in configuration and in direction, degree and duration (less than 1.2 seconds) of change. Many swallows were not followed by pressure complexes in the cervical esophagus, but when these did occur, they were of normal configuration and amplitude.

Radiologic appearance and correlation with pressure changes (Figures 2 and 3 ). Essentially similar findings were obtained in all three patients. The tongue apparently functioned normally, propelling the bolus back into the pharynx. However, the soft palate never closed off the nasal passages, presumably one reason why pressure could not be generated in the upper part of the pharynx. The barium, once introduced into the pharynx, ran down the posterior aspect of the tongue into the valleculae and down the pyriform sinuses. Elevation of the larynx and closure of the glottis were seen but appeared late and only when the barium bolus was well into the pharynx. As the larynx rose, the cricopharyngeus opened to allow the barium to pass on into the esophagus. Following the abrupt closure of the cricopharyngeus, a resi- 


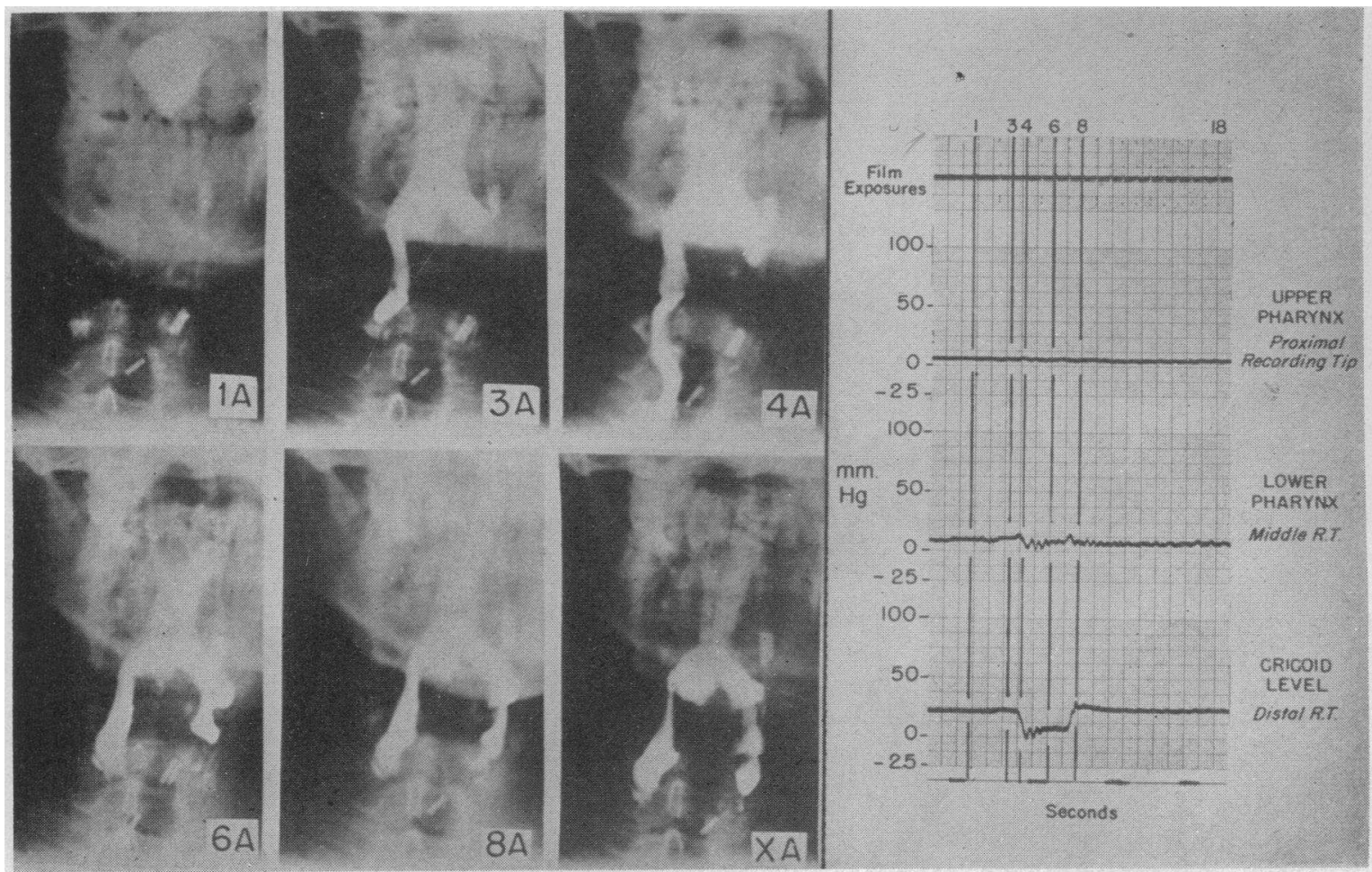

Fig. 2. Simultaneous Radiographic and Intraluminal Pressure Studies in a Patient with Dysphagia Following Poliomyelitis

Plate XA was taken 10 seconds after swallowing and shows the barium residue in the pharynx.

due of barium was left in the valleculae and pyriform sinuses.

Comment. That the patient with dysphagia following bulbar poliomyelitis can develop little if any change in intrapharyngeal pressure on swallowing has been noticed in one case previously reported from this laboratory (7). This finding is in keeping with the extensive paralysis of the pharyngeal constrictor muscles and the muscles of the soft palate which is frequently found following bulbar poliomyelitis. It is remarkable that in the presence of this extensive paralysis of the pharyngeal constrictors the cricopharyngeus should remain intact and function normally. Sparing of the cricopharyngeus, however, has been noted radiologically in other bulbar disorders which have caused pharyngeal constrictor weakness (8). Possibly the differential effect of bulbar poliomyelitis on constrictors and cricopharyngeus is related to the innervation of the pharyngoesophageal area. The muscles of the upper pharynx are supplied directly from motor cells in the nucleus ambiguus, through the ninth and tenth cranial nerves. The esophagus derives its parasympathetic (motor) supply from the dorsal nucleus of the vagus via preganglionic fibers running in the vagus nerve. At what point in the gullet the dorsal nucleus takes over the motor nerve supply from the nucleus ambiguus, or to what extent these supplies overlap, is uncertain although Mitchell states that the field supplied by the dorsal nucleus extends as high as the lower pharynx (9).

Poliomyelitis involves the nucleus ambiguus much more frequently than it does the dorsal nucleus of the vagus, and Faber and Silverberg (10) could discover no evidence of damage to the latter at autopsy in patients with bulbar poliomyelitis. Our finding of sparing of the cricopharyngeus after bulbar poliomyelitis might thus be explained on the grounds that this area, unlike the pharyngeal constrictor muscles, is adequately supplied by nerves from the dorsal nucleus. Functionally, then, this sphincter behaves as part of the esophagus rather than the pharynx.

Although flaccid paralysis of the upper esopha- 


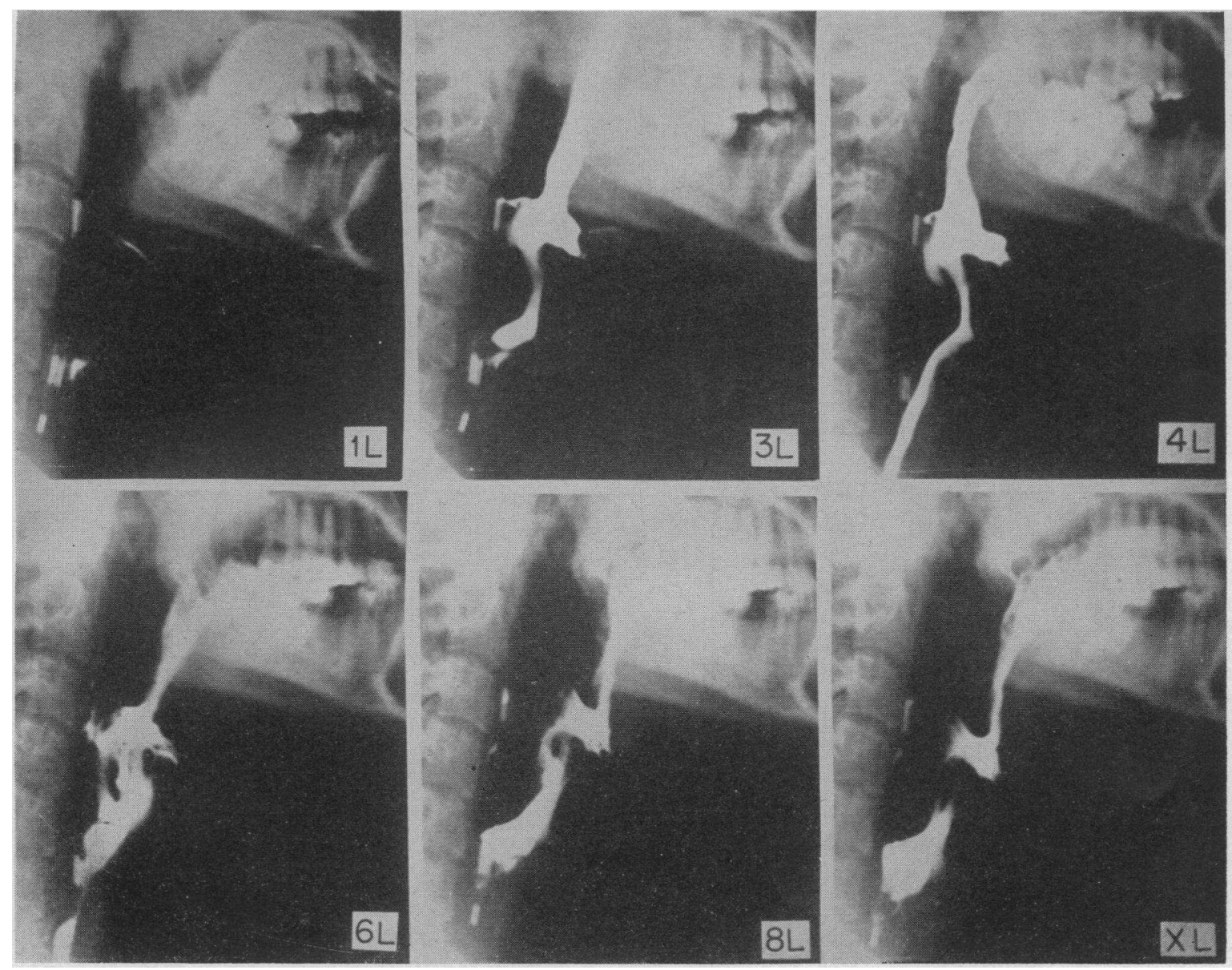

Fig. 3. Dysphagia Following Poliomyelitis

Lateral plates exposed at the same times as the anterop ssterior films shown in Figure 2.

gus has been stated to occur in bulbar lesions (11), the evidence is not clear. The upper esophagus appeared normal in our patients, and normal swallowing pressures occurred, albeit infrequently. These observations are in keeping with the finding of normal complexes in the mid- and distal esophagus of a bulbar poliomyelitis patient with total dysphagia (7). It would appear that little disturbance of intrinsic esophageal motility is present in patients with dysphagia following bulbar poliomyelitis.

In the patients here reported, a major part of each bolus passed into the esophagus with the aid of gravity, even in the absence of normal propulsive forces. Some barium remained in the pharynx because the flaccid pharyngeal walls and absence of constrictor muscle activity allowed pools to remain in the pyriform sinuses and valle- culae. Although the slow trickle of barium from the pharynx into the esophagus was interrupted by the closure of the cricopharyngeus, it seemed unlikely that a great deal more barium would have left the pharynx had this sphincter remained open. Certainly our findings failed to show any evidence of cricopharyngeal achalasia which has been described after poliomyelitis (4) or of spasm of the cricopharyngeus. Section of the cricopharyngeus has been used in the treatment of dysphagia following poliomyelitis (12). In light of our observations it seems doubtful whether this operation has any rational basis. We found no evidence of abnormal function of the cricopharyngeus, and the muscle can be relaxed again by taking a second "dry" swallow to allow the passage of any of the bolus that sphincteric closure might have prevented. 


\section{Myasthenia gravis}

Two patients with myasthenia gravis were studied immediately before and 20 minutes after their regular dose of prostigmine was given.

Resting intraluminal pressures. Pressures in the lumen of the pharynx and esophagus were within normal limits. At the level of the cricoid cartilage, in contrast to the group with dysphagia following poliomyelitis, both patients showed a low resting pressure. In one patient, no elevation could be detected at all (Figure 4), and in the other, the maximum resting pressure attained was $15 \mathrm{~mm} . \mathrm{Hg}$. After prostigmine the resting pressure rose to $10 \mathrm{~mm}$. $\mathrm{Hg}$ above the esophageal level in the first patient (Figure 4). In the second, the maximum resting pressure showed no change.

Deglutition pressures (Figure 4). On swallowing, both patients showed two very low peaks of intrapharyngeal pressure, corresponding in time with those seen in normal subjects. At the cricoid level, no pressure changes occurred in the patient who had no elevation of resting pressure; in the other patient, the swallowing complex was normal in configuration but slight in degree.

Radiologic appearance and correlation with pressure changes (Figure 5). The patients appeared to initiate swallowing by allowing the barium to run off the tongue into the pharynx. The normal thrusting action of the tongue by its approximation against the hard palate was not seen. As was true with the group with dysphagia following poliomyelitis, the barium had passed well into the pharynx before the larynx was elevated. In both subjects, as judged radiologically, the nasopharynx was closed off and weak constrictor muscle contractions occurred. In the patient with the high resting pressure at the cricopharyngeal area, the sphincter appeared to function normally.

After prostigmine, the thrusting action of the tongue returned sufficiently to give an impetus to the bolus and to cause a larger initial peak in the upper and lower pharyngeal records. The second peak corresponding with constrictor activity also showed a slight increase in amplitude. No change in cricopharyngeal activity was detectable radiologically, although the pressure records showed some return towards normal pattern.

Comment. The muscular disorder in the two patients with myasthenia gravis was more general-

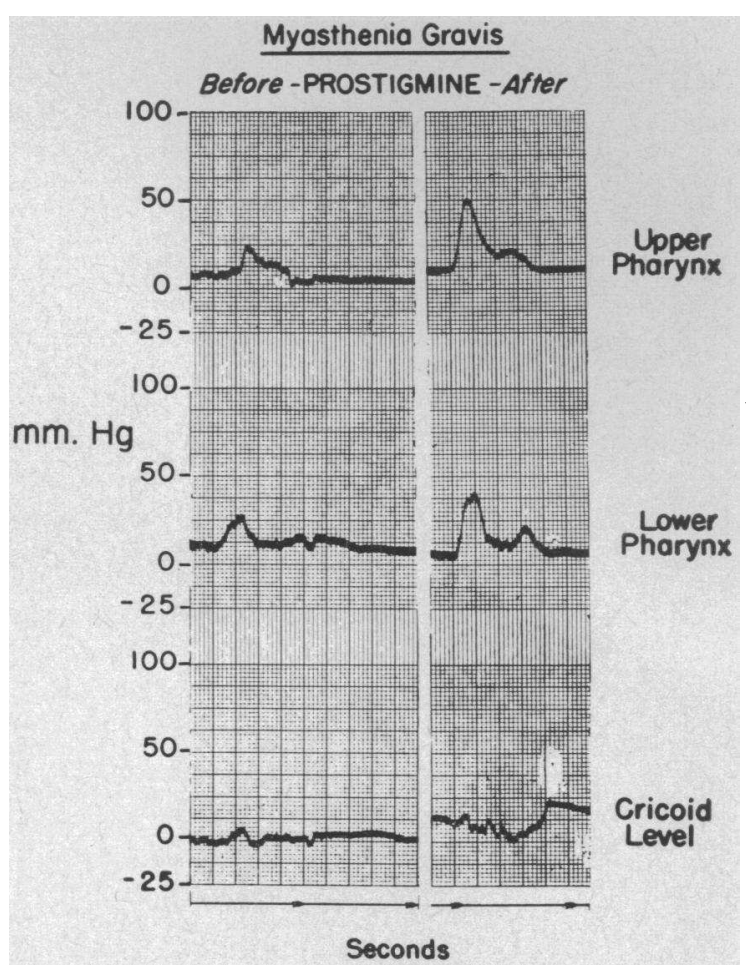

Fig. 4. Intraluminal Pressure Records from a Patient with Myasthenia Gravis Showing an InCrease in Amplitude After Prostigmine Injection

ized than that which followed bulbar poliomyelitis. In addition to the pharyngeal constrictor muscles, the tongue and cricopharyngeus were weakened and may have contributed to the difficulty in swallowing. Nevertheless, in the two patients studied the palatal muscles appeared to retain sufficient function to close off the nasal passages tightly enough to allow some pressure to build up in the pharynx. Since myasthenia gravis is a disease of striated muscle, involvement of the cricopharyngeus would be expected.

Our finding of increased intrapharyngeal deglutition pressures after prostigmine is in keeping with the improvement in swallowing noted radiographically after prostigmine by Schwab and Viets (13). They observed a reduction in the pharyngeal residue of barium in 17 out of 19 myasthenics given $1.5 \mathrm{mg}$. of prostigmine subcutaneously.

\section{Dystrophia myotonica}

Resting intraluminal pressures. Resting intraluminal pressures were in the normal range except 


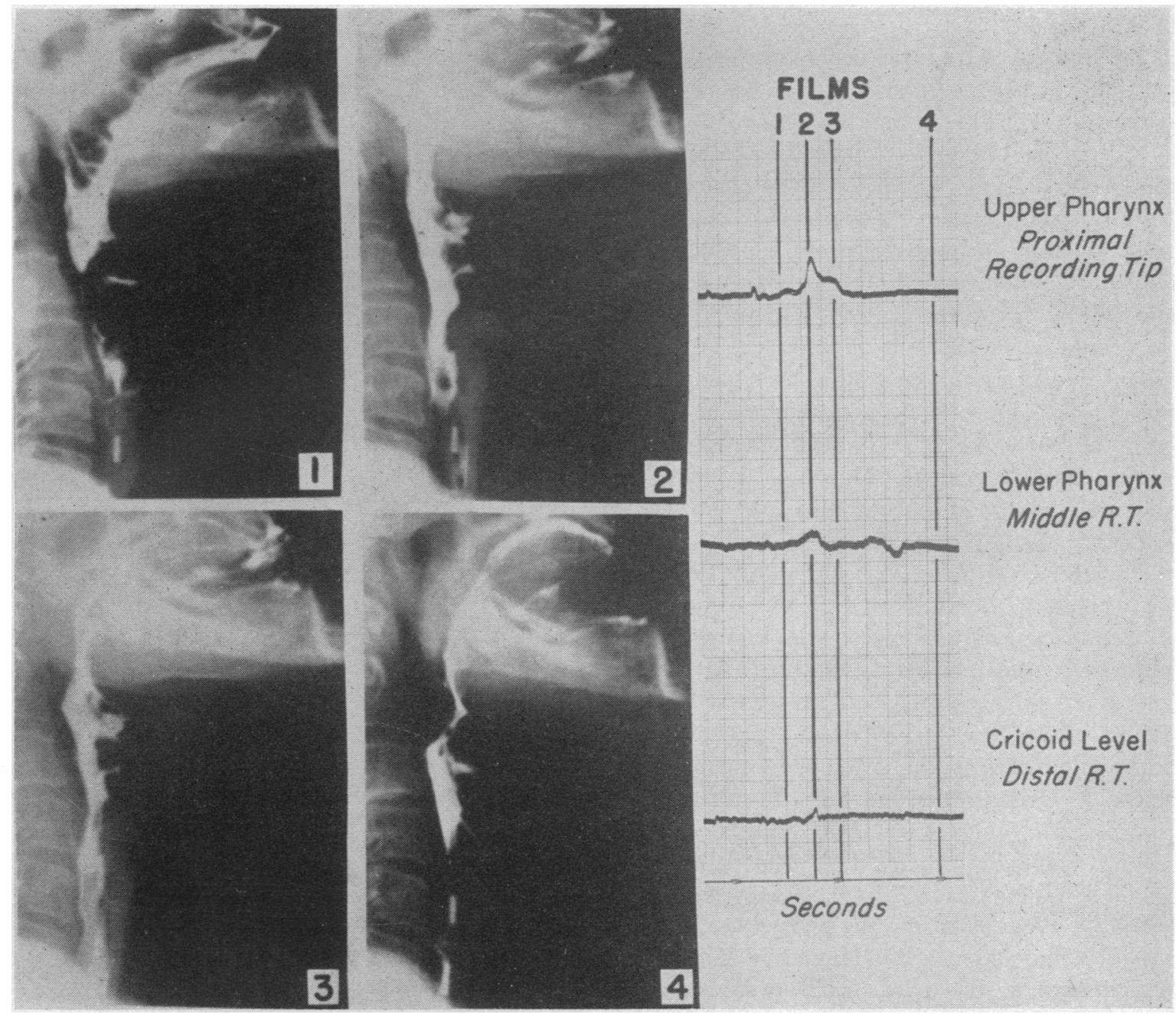

Fig. 5. Simultaneous Radiographic and Manometric Studies in a Patient with Myasthenia Gravis

for the cricopharyngeal area, where the normal elevation of resting pressure was not detected. In addition, the recording tip could be passed back and forth from pharynx into the esophagus without encountering a sense of resistance.

Deglutition pressures. Virtually no change in intraluminal pressures occurred on swallowing either in the pharynx, the cricopharyngeal zone or the upper esophagus.

Radiologic appearance and correlation with pressure changes. The patient had extensive involvement of the lingual muscles and could initiate swallowing only by tilting his head backwards and thus allowing the bolus to run into the pharynx. Radiologically, the thrusting action of the tongue was not seen and the barium appeared to run passively off the back of the tongue. It then trickled slowly through the pharynx. The nasal passages did not appear to be closed off, and very little elevation of the larynx could be observed. After swallowing, a considerable residue of barium remained in the recesses of the flaccid pharynx.

Comment. The swallowing disorder in this patient resembled that seen in myasthenia gravis in that all the muscle groups involved in the swallowing act appeared to be involved.

\section{DISCUSSION}

This study indicates that a number of factors may be responsible for difficulty in swallowing in patients with neuromuscular dysphagia. In nor- 
mal swallowing the impetus given to the bolus by the action of the tongue pressing against the hard palate appears to be of great importance in propelling the bolus through the pharynx and esophagus. In neuromuscular dysphagia this impetus is often absent because of either paralysis of tongue muscles themselves or inability to close off the nasal passages, with dissipation of pressure and possibly regurgitation through the nose. Under these circumstances the bolus passes into the pharynx largely by the action of gravity, its speed is much reduced, and swallowing is often impossible in the supine position. The head of the bolus appears to pass well over the back of the tongue into the valleculae before the involuntary phase of swallowing begins. In relation to the first voluntary instigation of swallowing, consequently, the involuntary functions of laryngeal elevation and closure and of cricopharyngeal opening may occur late. Because of the slow rate of flow of the bolus, however, this delay is probably more beneficial than otherwise. The cricopharyngeus still opens in advance of arrival of the bolus, and closing of the respiratory passages is postponed until the last moment, thus shortening the long respiratory interruption necessitated by the slow passage of swallowed material.

Residues of barium are seen in the valleculae and pyriform sinuses in the presence of paralysis of the pharyngeal constrictors irrespective of whether the cricopharyngeus is functioning normally or is paralyzed. These residues therefore appear to be due to failure of the action of the constrictor muscles in emptying these recesses.

\section{SUMMARY}

Disorders of swallowing in four patients with post-poliomyelitis dysphagia, two with myasthenia gravis and one with dystrophia myotonica have been studied by the recording of intraluminal pressures from the pharynx and esophagus combined with synchronous rapid serial radiography during barium swallows.

After poliomyelitis, failure to close off the nasal passages, with consequent dissipation of oropharyngeal pressure, and paralysis of the pharyngeal constrictor muscles appeared to be the principal abnormalities. The cricopharyngeus functioned normally in each of the four patients, and no evidence of achalasia or spasm was obtained.
In two patients with myasthenia gravis and one with dystrophia myotonica, a generalized muscular weakness, particularly involvement of the tongue muscles, appeared to be important in producing dysphagia. The cricopharyngeus, a striated muscle, was involved.

\section{ACKNOWLEDGMENTS}

We are grateful to Dr. Louis Weinstein of the Haynes Memorial Hospital, Boston, and to Dr. Vincent P. Perlo of the Massachusetts General Hospital, Boston, for making their patients available to us for study.

\section{REFERENCES}

1. McGibbon, J. E. G., and Mather, J. H., Vallecular dysphagia. Brit. M. J., 1933, II, 1013.

2. Brunner, $H$., The cricopharyngeal muscle under normal and pathological conditions. Arch. Otolaryng., 1952, 56, 616.

3. Montandon, A., Pseudo-spasme permanent de la bouche de l'oesophage et myoporphyrie. Pract. oto-rhino-laryng., 1948, 10, 267.

4. Asherson, N., Achalasia of the cricopharyngeal sphincter: A record of cases, with profile pharyngograms. J. Laryng. \& Otol., 1950, 64, 747.

5. Atkinson, M., Kramer, P., Wyman, S. M., and Ingelfinger, F. J., The dynamics of swallowing. I. Normal pharyngeal mechanisms. J. Clin. Invest., 1957, 36, 581.

6. Fyke, F. E., and Code, C. F., Resting and deglutition pressures in the pharyngo-esophageal region. Gastroenterology, 1955, 29, 24.

7. Kramer, P., Minkel, H., Frank, H., and Ingelfinger, F. J., Normal and abnormal swallowing mechanisms studied by the measurement of intraluminal esophagus pressures. J. Clin. Invest., 1951, 30, 653.

8. Templeton, F. E., X-ray Examination of the Stomach. Chicago, University of Chicago Press, 1944.

9. Mitchell, G. A. G., Anatomy of the Autonomic Nervous System. Edinburgh, Livingstone, 1953.

10. Faber, H. K., and Silverberg, R. J., A neuropathological study of acute human poliomyelitis with special reference to the initial lesion and to various potential portals of entry. J. Exper. Med., 1946, 83, 329.

11. Otell, L. S., and Coe, F. O., Dysphagia-roentgenologically considered. Am. J. Digest. Dis., 1935, 2, 117.

12. Kaplan, S., Paralysis of deglutition, a post-poliomyelitis complication treated by section of the cricopharyngeus muscle. Ann. Surg., 1951, 133, 572.

13. Schwab, R. S., and Viets, H. R., Roentgenoscopy of the pharynx in myasthenia gravis before and after prostigmine injection. Am. J. Roentgenol., 1941, 45, 357. 